\title{
A New Transport Interaction of Dibasic Amino Acids and Gitrulline in Human Kidney
}

\author{
Kazuhiko Oyanagi, Hideaki Sogawa, RyoJi Minami, \\ Tooru NakaO, Kooji Karube* and Satoshi Tsugawa* \\ Department of Pediatrics, Sapporo Medical College, Sapporo \\ 060 and *Department of Pediatrics, Otaru National Hospital, \\ Otaru $04 \%$
}

Oyanagi, K., Sogawa, H., Minami, R., Nakao, T., Karube, K. and Tsugawa, S. A New Transport Interaction of Dibasic Amino Acids and Citrulline in Human Kidney. Tohoku J. exp. Med., 1981, 134 (1), 55-58—The oral loading tests of lysine in 9 healthy men and the intravenous loading tests of lysine, ornithine and arginine in 3 healthy men were carried out. The results indicated that the membrane transport system of citrulline in the human kidney was clearly inhibited by dibasic amino acids, lysine, ornithine, and arginine. - dibasic amino acids; citrulline; membrane transport

Cystinuria is a well-known transport disorder of cystine and the dibasic amino acids, lysine, ornithine, and arginine. Dent and Rose (1951) first postulated that reabsorption of these four amino acids in the renal tubule cell is defective in cystinuric patients. Recent studies (Rosenberg et al. 1962; Fox et al. 1964; Segal et al. 1977) using both rat and human kidney cortex slices revealed that dibasic amino acids shared a common transport system, but that cystine uptake was controlled by an independent mechanism.

Several clinical observations, of hyperdibasicaminoaciduria without cystinuria (Oyanagi et al. 1970; Whelan and Scriver 1968) and cystinuria without hyperdibasicaminoaciduria (Brodehl et al. 1967), support separate membrane transport systems for cystine and dibasic amino acids.

The purpose of the present study is to describe the transport interaction of dibasic amino acids and citrulline which was hitherto unrecognized in the human kidney.

\section{SubJects ANd Methods}

Nine healthy colleagues of our Department participated in the following experiments.

Oral loading tests of L-lysine. After overnight fasting, $150 \mathrm{mg}$ of L-lysine per $\mathrm{kg}$ of body weight were administered orally. Urine specimens were collected before and after the loading for $4 \mathrm{hr}$. Quantitative analyses of amino acids were performed by a Technicon Amino-Acid Auto Analyzer.

Constant infusion tests with L-lysine, L-ornithine, and L-arginine. $12 \mathrm{ml} / \mathrm{kg}$ body weight/hr of $20 \mathrm{mM}, 40 \mathrm{mM}$, and $80 \mathrm{mM} \mathrm{L}$-lysine, L-ornithine, and L-arginine solutions were constantly infused for $90 \mathrm{~min}$ on separate days, and blood samples were taken before

Received for publication, July 21, 1980. 
and just after the load. Urine specimens were collected for $30 \mathrm{~min}$ during the final 30 min of the load. Amino acids in serum and urine were measured by means of an automatic amino acid analyzer (Hitachi, KLA 5).

\section{RESULts}

The results obtained are shown in Tables 1,2 and 3. The urinary excretion rates of lysine after the oral loading of L-lysine increased 1.5 times as compared with those before the loading, whereas cystine and other dibasic amino acids were unchanged. On the other hand, the urinary excretion rates of citrulline after the loading were clearly elevated, with these findings being observed in all urine specimens tested. However, the urinary excretion rates of other neutral amino acids after the loading were slightly decreased (Table 1).

The urinary excretion rates of citrulline during constant infusion of dibasic amino acids were gradually increased according to the concentration of infused amino acids as were those of cystine and dibasic amino acids (Table 2). On the

TABLE 1. Urinary excretion rates of amino acids before and after loading of lysine, $150 \mathrm{mg} / \mathrm{kg}$

\begin{tabular}{lrrrrr}
\hline & \multicolumn{2}{c}{ Before loading $(n=9)$} & & \multicolumn{2}{c}{ After loading $(n=9)$} \\
\cline { 2 - 3 } \cline { 5 - 6 } Amino acid & Mean & Range & & Mean & Range \\
\hline Threonine & 291 & $120-483$ & & 250 & $147-484$ \\
Serine & 509 & $345-754$ & & 442 & $279-660$ \\
Glutamic acid & 169 & $87-325$ & & 156 & $88-246$ \\
Glycine & 1335 & $554-2640$ & & 1059 & $388-1952$ \\
Alanine & 318 & $115-579$ & & 304 & $121-640$ \\
Valine & 54 & $36-90$ & & 33 & $25-48$ \\
Cystine & 148 & $65-283$ & & 120 & $73-247$ \\
Methionine & 27 & trace- 46 & & 20 & $2-40$ \\
Citrulline & 19 & trace- 43 & & 101 & $63-165$ \\
Isoleucine & 47 & $17-127$ & & 33 & $21-52$ \\
Leucine & 55 & $31-90$ & & 56 & $43-$ \\
Tyrosine & 104 & $62-192$ & & 96 & $57-141$ \\
Phenylalanine & 66 & $39-131$ & & 55 & $35-76$ \\
Ornithine & 39 & $17-66$ & & 38 & $19-72$ \\
Lysine & 942 & $231-1741$ & & 1418 & $184-3220$ \\
Histidine & 1095 & $675-1775$ & & 1014 & $638-1313$ \\
Arginine & 28 & $12-56$ & 31 & $13-83$ \\
\hline
\end{tabular}

Values are expressed as $\mu$ moles/g creatinine.

TABLE 3. Serum concentrations

\begin{tabular}{|c|c|c|c|c|c|}
\hline & \multicolumn{4}{|c|}{ Lysine infusion } & \multirow{2}{*}{$\begin{array}{c}\text { Ornithine } \\
\text { Before }\end{array}$} \\
\hline & Before & $20 \mathrm{mM}$ & $30 \mathrm{mM}$ & $80 \mathrm{mM}$ & \\
\hline \multicolumn{6}{|c|}{$\begin{array}{l}\text { Serum citrulline } \\
(\mu \text { moles/liter })\end{array}$} \\
\hline Case 1 & 13 & 9 & 9 & 9 & 6 \\
\hline Case 2 & 24 & 12 & 16 & 12 & 13 \\
\hline Case 3 & 21 & 14 & 14 & 11 & 6 \\
\hline
\end{tabular}


TABLE 2. Urinary excretion rates of dibasic amino acids, cystine and citrulline before and after constant infusion

\begin{tabular}{|c|c|c|c|c|c|c|c|c|c|c|}
\hline \multirow{2}{*}{ Amino } & \multirow{2}{*}{ acids } & \multicolumn{3}{|c|}{ Lysine infusion } & \multicolumn{3}{|c|}{ Ornithine infusion } & \multicolumn{3}{|c|}{ Arginine infusion } \\
\hline & & Case 1 & Case 2 & Case 3 & Case 1 & Case 2 & Case 3 & Case 1 & Case 2 & Case 3 \\
\hline Lysine & $\begin{array}{l}\text { Before } \\
20 \mathrm{mM} \\
40 \mathrm{mM} \\
80 \mathrm{mM}\end{array}$ & $\begin{array}{r}53 \\
18800 \\
134300 \\
421000\end{array}$ & $\begin{array}{r}82 \\
13700 \\
94300 \\
404700\end{array}$ & $\begin{array}{r}83 \\
5041 \\
92940 \\
410000\end{array}$ & $\begin{array}{r}435 \\
4585 \\
12115 \\
20330\end{array}$ & $\begin{array}{r}126 \\
2429 \\
11600 \\
22120\end{array}$ & $\begin{array}{r}37 \\
1870 \\
9564 \\
22375\end{array}$ & $\begin{array}{r}976 \\
7949 \\
9194 \\
14370\end{array}$ & $\begin{array}{r}457 \\
4493 \\
9892 \\
16970\end{array}$ & $\begin{array}{r}140 \\
4071 \\
19236 \\
26500\end{array}$ \\
\hline Ornithine & $\begin{array}{l}\text { Before } \\
20 \mathrm{mM} \\
40 \mathrm{mM} \\
80 \mathrm{mM}\end{array}$ & $\begin{array}{r}53 \\
336 \\
2167 \\
3618\end{array}$ & $\begin{array}{r}66 \\
173 \\
977 \\
2753\end{array}$ & $\begin{array}{r}169 \\
70 \\
1210 \\
3567\end{array}$ & $\begin{array}{r}21 \\
5894 \\
65920 \\
270790\end{array}$ & $\begin{array}{r}49 \\
1271 \\
33670 \\
214650\end{array}$ & $\begin{array}{r}102 \\
1607 \\
46180 \\
420750\end{array}$ & $\begin{array}{r}81 \\
7205 \\
34730 \\
121500\end{array}$ & $\begin{array}{r}202 \\
578 \\
6432 \\
28250\end{array}$ & $\begin{array}{r}95 \\
657 \\
23444 \\
82738\end{array}$ \\
\hline Arginine & $\begin{array}{l}\text { Before } \\
20 \mathrm{mM} \\
40 \mathrm{mM} \\
80 \mathrm{mM}\end{array}$ & $\begin{array}{r}5 \\
451 \\
4286 \\
17590\end{array}$ & $\begin{array}{r}29 \\
236 \\
2081 \\
8414\end{array}$ & $\begin{array}{r}6 \\
77 \\
2559 \\
11027\end{array}$ & $\begin{array}{r}5 \\
380 \\
4098 \\
4829\end{array}$ & $\begin{array}{r}6 \\
143 \\
3084 \\
11060\end{array}$ & $\begin{array}{r}5 \\
115 \\
3282 \\
12663\end{array}$ & $\begin{array}{r}12 \\
3881 \\
31980 \\
168300\end{array}$ & $\begin{array}{r}202 \\
1671 \\
32160 \\
207720\end{array}$ & $\begin{array}{r}2 \\
1086 \\
93819 \\
447280\end{array}$ \\
\hline Cystine & $\begin{array}{l}\text { Before } \\
20 \mathrm{mM} \\
40 \mathrm{mM} \\
80 \mathrm{mM}\end{array}$ & $\begin{array}{r}85 \\
316 \\
1126 \\
3090\end{array}$ & $\begin{array}{r}72 \\
297 \\
904 \\
2897\end{array}$ & $\begin{array}{r}41 \\
25 \\
1108 \\
2395\end{array}$ & $\begin{array}{r}121 \\
606 \\
1738 \\
2842\end{array}$ & $\begin{array}{r}44 \\
129 \\
1216 \\
2831\end{array}$ & $\begin{array}{r}75 \\
261 \\
1346 \\
3500\end{array}$ & $\begin{array}{r}271 \\
1898 \\
2484 \\
3493\end{array}$ & $\begin{array}{r}195 \\
694 \\
3027 \\
4277\end{array}$ & $\begin{array}{r}51 \\
1286 \\
4347 \\
5430\end{array}$ \\
\hline Citrulline & $\begin{array}{l}\text { Before } \\
20 \mathrm{mM} \\
40 \mathrm{mM} \\
80 \mathrm{mM}\end{array}$ & $\begin{array}{r}3 \\
25 \\
154 \\
554\end{array}$ & $\begin{array}{r}9 \\
37 \\
193 \\
1069\end{array}$ & $\begin{array}{r}7 \\
10 \\
320 \\
1407\end{array}$ & $\begin{array}{r}8 \\
56 \\
262 \\
868\end{array}$ & $\begin{array}{r}6 \\
343 \\
463 \\
857\end{array}$ & $\begin{array}{r}6 \\
149 \\
603 \\
1575\end{array}$ & $\begin{array}{r}16 \\
119 \\
210 \\
880\end{array}$ & $\begin{array}{r}28 \\
144 \\
865 \\
1295\end{array}$ & $\begin{array}{r}9 \\
128 \\
500 \\
2075\end{array}$ \\
\hline
\end{tabular}

Values are expressed as $\mu$ moles/g creatinine.

other hand, the serum concentrations of citrulline during infusion were unchanged (Table 3).

\section{Discussion}

Citrulline is one of the neutral amino acids and it is generally believed that amino acid groups in the human kidney, such as neutral amino acid, acidic amino acid, cystine and dibasic amino acids, glycine and imino acids, and $\beta$-amino acids, apparently share a common transport system (Rosenberg et al. 1962; Wilson and Scriver 1967; Scriver and Wilson 1967). The inborn errors of amino acid transport support the separate membrane transport systems.

The present study, the oral loading tests and constant infusion tests of dibasic amino acids in healthy men, revealed the existence of a transport interaction in

of citrulline during constant infusion

\begin{tabular}{cccccccc}
\hline infusion & & & \multicolumn{5}{c}{ Arginine infusion } \\
\hline $20 \mathrm{mM}$ & $40 \mathrm{mM}$ & $80 \mathrm{mM}$ & & Before & $20 \mathrm{mM}$ & $40 \mathrm{mM}$ & $80 \mathrm{mM}$ \\
\hline & & & & & & \\
6 & 6 & 5 & 4 & 4 & 4 & 7 \\
40 & 18 & 4 & 5 & 5 & 5 & 4 \\
6 & 6 & 5 & 5 & 5 & 5 & 4 \\
\hline
\end{tabular}


the human kidney between dibasic amino acids and citrulline which is not as yet recognized.

It is difficult to explain the existence of this transport system, but it may be speculated that citrulline has a structural resemblance to dibasic amino acids.

\section{References}

1) Brodehl, J., Gellissen, K. \& Kowalewski, S. (1967) Isollierte Cystinurie in einer Familie mit hypocalcemischer Tetanie. Mschr. Kinderheilk., 115, 317-320.

2) Dent, C.E. \& Rose, G.A. (1951) Amino acid metabolism in cystinuria. Quart. J. Med., 20, 205.

3) Fox, M., Their, S., Rosenberg,L.E., Kiser, W. \& Segal, S. (1964) Evidence against a single renal transport defect in cystinuria. New. Engl. J. Med., 270, 556-561.

4) Oyanagi, K., Miura, R. \& Yamanouchi, T. (1970) Congenital lysinuria, a new inherited transport disorder of dibasic amino acids. J. Pediat., 77, 259-266.

5) Rosenberg, L.E., Dowing, S.J. \& Segal, S. (1962) Competitive inhibition of dibasic amino acid transport in rat kidney. J. biol. Chem., 237, 2265-2270.

6) Scriver, C.R. \& Wilson, O.H. (1967) Amino acid transport: Evidence for genetic control of two types in human kidney. Science, 155, 1428-1430.

7) Segal, S., McNamara, P.D. \& Pepe L.M. (1977) Transport interaction of cystine and dibasic amino acids in renal brush border vesicles. Science, 197, 169-171.

8) Whelan, D.T. \& Scriver, C.R. (1968) Hyperdibasicaminoaciduria: An inherited disorder of amino acid transport. Pediat. Res., 2, 525-534.

9) Wilson, O.H. \& Scriver, C.R. (1967) Specificity of transport of neutral and basic amino acids in rat kidney. Amer. J. Physiol., 213, 185-190. 